\title{
Efecto del enriquecimiento ambiental en un grupo de monos araña (Ateles geoffroyi) en cautiverio
}

\author{
Alejandra Márquez-Arias, ${ }^{1}$ Ana María Santillán-Doherty, ${ }^{2}$ Rita Virginia Arenas-Rosas, ${ }^{2}$ \\ Marco Polo Gasca-Matías, ${ }^{2}$ Jairo Muñoz-Delgado, ${ }^{2,3}$ Javier Villanueva-Valle²
}

Artículo original

\section{SUMMARY}

Captive animals suffer from stress and lack of stimulation. Environmental enrichment (EE) provides them with a series of activities that encourages them to present species-specific behaviors. The objective of this work was to analyze the effect of an EE program in the behavior of a 7-spider monkey colony. It was expected that aggression, coprophilia and stereotypes would be less frequent, while exploration and play would increase. The study was divided in two stages: one previous to $\mathrm{EE}$ and one while EE was being implemented. A focal sampling per behavior was undertaken in 1 -h continuous recordings for a total of 40 hours per stage. We compared the frequency of each behavior with a Wilcoxon's signal test. The results showed that aggression, stereotypes and coprophilia were reduced during EE, while exploration and play increased significantly. No significant differences were found between sexes. EE reduces the problems originated by confinement; therefore, it must be considered a necessary tool to be continuously employed to make an improvement into the life conditions of confined individuals.

Key words: Behavioral change, animal welfare, environmental enrichment, Ateles geoffroyi.

\section{RESUMEN}

Los animales que viven en cautiverio padecen problemas relacionados con el estrés y la falta de estimulación. El enriquecimiento ambiental (EA) proporciona a los animales en esta situación un conjunto de actividades que los alienta a presentar conductas típicas de su especie. El objetivo de este trabajo fue analizar el efecto de un programa de EA en la conducta de una colonia de siete monos araña (Ateles geoffroyi). Se esperaba que la agresión, la coprofilia y las estereotipias fueran menos frecuentes entre los monos, mientras que la exploración y el juego se presentaran con mayor frecuencia. Para ello, la investigación se dividió en dos etapas: una etapa previa al EA y una etapa durante el EA. En cada etapa se registraron las siguientes categorías conductuales: agresión, coprofilia, estereotipias, exploración y juego. Se utilizó un muestreo focal por conducta en registros continuos de $1 \mathrm{hr}$., obteniéndose en cada etapa $40 \mathrm{hrs}$. de observación. Al comparar la frecuencia de dichas conductas con la prueba de los signos de Wilcoxon, se encontró que la agresión, las estereotipias y la coprofilia disminuyeron durante el EA. La exploración y el juego se incrementaron significativamente con el EA. No se observaron diferencias significativas al comparar el sexo de los individuos. El EA disminuye los problemas causados por el confinamiento, por lo que debe contemplarse como una herramienta indispensable que de manera constante mejore las condiciones de vida en individuos confinados.

Palabras clave: Cambio conductual, bienestar animal, enriquecimiento ambiental, Ateles geoffroyi.

\section{INTRODUCCIÓN}

El cautiverio provoca graves cambios en el comportamiento de los animales. Tanto en primates humanos y no humanos se observa un incremento en la frecuencia de conductas agresivas hacia otros individuos y hacia ellos mismos, ${ }^{1,2}$ se desarrollan conductas estereotipadas autodirigidas como jalarse el cabello, jalarse las extremidades y caminar en forma de círculo. ${ }^{2-4}$
Además de lo antes mencionado, en primates no humanos se observa específicamente una mayor inactividad, menos conductas de locomoción y de juego, y los periodos de sueño son mayores en comparación con animales de la misma especie que viven en libertad. ${ }^{5-7}$ Asimismo, se sabe que las conductas agresivas, estereotipadas y coprofílicas son indicadoras del grado de estrés o aburrimiento, ${ }^{8,9}$ mientras que conductas como la exploración y el juego se consideran indicadoras del bienestar de los individuos..$^{10}$

Universidad Nacional Autónoma de México, UNAM

Laboratorio de Cronoecología y Etología Humana. Dirección de Neurociencias. Instituto Nacional de Psiquiatría Ramón de la Fuente Muñiz.

Facultad de Psicología, UNAM.

Correspondencia: Ana María Santillán-Doherty. Laboratorio de Cronología y Etología Humana. Dirección de Neurociencias, Instituto Nacional de Psiquiatría Ramón de la Fuente Muñiz. Calz. México-Xochimilco 101, San Lorenzo Huipulco, Tlalpan, 14370, México, DF. Fax: 5655 - 9980. E-mail: santild@gmail.com 
El enriquecimiento ambiental (EA) es un conjunto de acciones que propician un entorno estimulante para los individuos que viven cautivos con el objeto de que su comportamiento sea semejante a los individuos de la misma especie que viven en vida libre, con lo que se procura el bienestar de los animales. ${ }^{11-14}$

En las últimas décadas, el EA ha tenido un auge importante para aplicarse entre los primates no humanos, ${ }^{2,15-17}$ ya que en los laboratorios es posible no sólo aminorar los efectos del confinamiento, ${ }^{18,19}$ sino además mejorar los resultados obtenidos en estudios de conductas aberrantes y las alternativas para disminuir éstas son útiles para la ciencia médica. ${ }^{1,320}$ Dado que en humanos con algún problema mental o en reclusión penitenciaria se presentan conductas similares a las de los primates no humanos en cautiverio, es pertinente implementar actividades que en este caso se conocen como de enriquecimiento ocupacional. ${ }^{21}$

En primates no humanos las estrategias aplicadas se pueden agrupar en tres tipos de EA según su objetivo. a. El enriquecimiento físico consiste en incrementar la complejidad en el ambiente, añadiendo estructuras en el suelo, en las paredes y en el techo, que permitan dividir el espacio en áreas funcionales. ${ }^{2,22,23}$ b. El enriquecimiento alimenticio incluye actividades que permiten que los monos prolonguen el tiempo y el esfuerzo que hacen para alimentarse, incluyendo el "forrajeo"; para ello se alternan los horarios y la forma en que los alimentos les son proporcionados., 2,13,22-24 c. La introducción de objetos novedosos tiene la finalidad de compensar la carencia de objetos manipulables dentro de los encierros. Para ello se proporcionan juguetes y otros objetos que pueden manipular los individuos., 2,13,22,23

Los beneficios reportados por el EA son numerosos. Por ejemplo, en humanos prisioneros se ha documentado que la estimulación mediante el ejercicio, el trabajo productivo e incluso la preparación de alimentos y el estudio, evitan los encuentros violentos. ${ }^{21}$

En monos araña se ha demostrado que el comportamiento se ve afectado por el tipo de encierro. Cardona-López et al. ${ }^{25}$ compararon el comportamiento de una colonia de Ateles fusciceps robustus que vivía en una jaula con otra colonia que vivía en condiciones de semilibertad. Los autores encontraron que los animales en cautiverio dedicaron más tiempo a descansar y a comer, en cambio los animales en semilibertad dedicaron más tiempo al acercamiento social y al desplazamiento. Además, el grupo que vivía enjaulado presentó más conductas estereotipadas que los animales que vivían en la isla.

Por su parte, Torstensson ${ }^{26}$ evalúo tres tipos de actividades de enriquecimiento alimenticio con monos arañas colombianos (A. fusciceps rufiventris) para conocer las actividades más atractivas para los monos, en función del tiempo que les dedicaban. El autor encontró que los monos prefieren la actividad de "rompecabezas de comida" por sobre las actividades de "bloques de hielo" y "pelotas de ramas". También describió mayor inactividad en los individuos cuando no se
Cuadro 1. Individuos de la colonia al momento del estudio

\begin{tabular}{lccc}
\hline Individuo & Sigla & Sexo & Categoría de edad \\
\hline Adrian & AD & Macho & Adulto \\
Canica & $\mathrm{Cl}$ & Hembra & Adulta \\
Celina & $\mathrm{CE}$ & Hembra & Adulta \\
Frida & $\mathrm{FD}$ & Hembra & Adulta \\
Kifir & $\mathrm{KI}$ & Macho & Adulto \\
Leakey & LK & Macho & Adulto \\
Lola & LO & Hembra & Adulta \\
\hline
\end{tabular}

aplican actividades de EA y los animales pierden interés en las actividades conforme se termina la comida involucrada en dicha actividad.

En el Instituto Nacional de Psiquiatría Ramón de la Fuente Muñiz de la Ciudad de México se encuentra una colonia de monos araña (A. geoffroyi) en que las estereotipias, las conductas agresivas y la coprofilia eran frecuentes. Además, los monos permanecieron más de 10 años en cautiverio sin un programa formal de EA, por lo que fue necesario ofrecerles una mejor calidad de vida mediante la aplicación de un Programa de Enriquecimiento Ambiental (PEA).

Se esperaba que con el EA disminuyera la frecuencia de las conductas agresivas, las estereotipias y la coprofilia, mientras que las conductas de exploración y juego se esperaba que fueran más frecuentes entre los monos. El objetivo de este trabajo fue evaluar el efecto del PEA integral aplicado a un grupo de monos araña (A. geoffroyi) en cautiverio.

\section{MATERIAL Y MÉTODO}

Al momento del estudio la colonia se componía de siete monos araña adultos: cuatro hembras y tres machos (cuadro 1).

Desde 1989 los monos forman una colonia y viven en cautiverio exterior en una jaula de forma cónica de seis me-
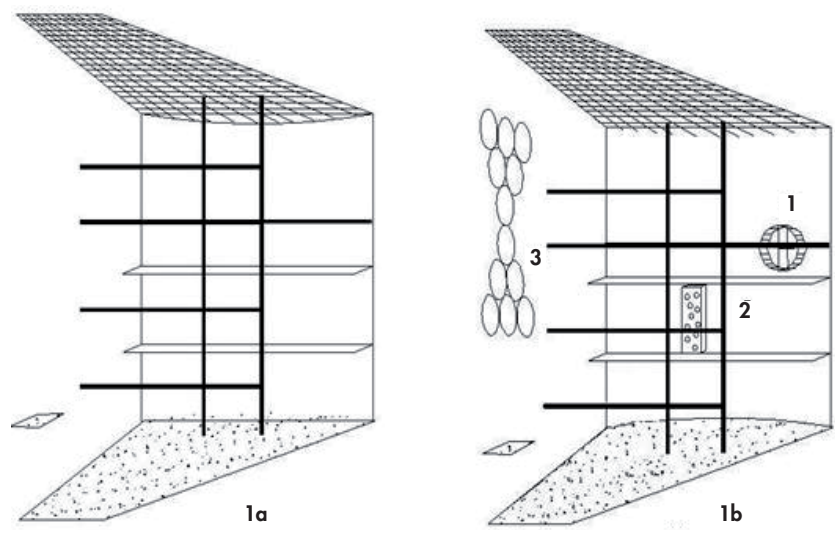

Figura 1. Esquema de la jaula. 1a. Condiciones de la jaula en la etapa inicial. $1 \mathrm{~b}$. Enriquecimiento físico: estructuras añadidas a la jaula como parte del enriquecimiento ambiental. 1. Rueda de ejercicio, 2. Panel de forrajeo, 3. Junga de aros. 
Cuadro 2. Actividades del Programa de Enriquecimiento Ambiental

\begin{tabular}{|c|c|}
\hline Actividad & Descripción \\
\hline Alimento en botes & $\begin{array}{l}\text { Se utilizaron botes de formula láctea marca Pro- } \\
\text { mil de } 800 \mathrm{gm} \text {. En la parte superior del bote se } \\
\text { formo una malla de alambre. Dentro de los botes } \\
\text { se colocó fruta y se dio a los monos para que } \\
\text { ellos sacaran el alimento. }\end{array}$ \\
\hline Alimento en costal & $\begin{array}{l}\text { La comida correspondiente a un día se colocó } \\
\text { dentro de un costal de yute amarrado. Posterior- } \\
\text { mente se dio a los monos. }\end{array}$ \\
\hline Alimento en techo & $\begin{array}{l}\text { La fruta correspondiente a un día se colocó en la } \\
\text { malla del techo de las jaulas. }\end{array}$ \\
\hline Botes con semillas & $\begin{array}{l}\text { En botes de aluminio que contenían jugo marca } \\
\text { JUMEX se introdujeron semillas de alpiste, de gi- } \\
\text { rasol, de trigo. Los botes con semillas se dieron } \\
\text { a los monos. }\end{array}$ \\
\hline Panel de forrajeo & $\begin{array}{l}\text { El panel de forrajeo es una lámina de metal con } \\
\text { orificios. Detrás de cada orificio hay una canas- } \\
\text { tilla de metal en la que se pueden colocar semi- } \\
\text { llas. Durante cada sesión con el panel de forra- } \\
\text { jeo se depositaron semillas de girasol, alpiste, } \\
\text { trigo, arroz, avena y cereal comercial. }\end{array}$ \\
\hline Pelotas & $\begin{array}{l}\text { Se utilizaron pelotas plásticas de } 30 \mathrm{~cm} \text {. de diá- } \\
\text { metro. Las pelotas se pusieron en la jaula de con- } \\
\text { tención que se había cerrado previamente, para } \\
\text { después abrirla y que los monos las sacaran. }\end{array}$ \\
\hline Ramas y hojas & $\begin{array}{l}\text { En la malla del techo de la jaula se proporciona- } \\
\text { ron ramas con hojas de Ficus sp. y Pinus sp. }\end{array}$ \\
\hline Rodillo & $\begin{array}{l}\text { El rodillo es un cilindro de metal de } 22 \mathrm{~cm} \text {. de } \\
\text { longitud } \times 11 \mathrm{~cm} \text {. de diámetro. Un tubo metáli- } \\
\text { co atraviesa el cilindro y de cada extremo del } \\
\text { cilindro tienen una saliente de } 10 \mathrm{~cm} \text {. De cada } \\
\text { lado del rodillo en la unión del cilindro con el } \\
\text { tubo se encuentra un balero que permite que el } \\
\text { rodillo ruede. }\end{array}$ \\
\hline Semillas & $\begin{array}{l}\text { A través de la malla del techo de las jaulas se es- } \\
\text { parcieron semillas de girasol, alpiste, trigo, arroz } \\
\text { y avena. Las semillas caían en el suelo de toda la } \\
\text { jaula y los monos podían tomarlas directamente } \\
\text { del suelo. }\end{array}$ \\
\hline Sonajas & $\begin{array}{l}\text { Unos tubos de cartón de } 4 \text { × } 10 \mathrm{~cm} \text {. se forraron } \\
\text { con papel periódico y pegamento hecho con hari- } \\
\text { na y agua. Dentro de estos cilindros se colocaron } \\
\text { semillas de girasol, alpiste, trigo, arroz y avena. }\end{array}$ \\
\hline $\begin{array}{l}\text { Troncos } \\
\text { con orificios }\end{array}$ & $\begin{array}{l}\text { Se utilizaron troncos de eucalipto a los que se } \\
\text { les hicieron pequeñas perforaciones en las que } \\
\text { se depositaron mermelada, leche condensada, } \\
\text { cajeta, salsa cátsup, mayonesa y miel. }\end{array}$ \\
\hline
\end{tabular}

tros de largo, 6.3 metros de alto, 6.2 metros de ancho en su parte mayor y 1.7 metros de ancho en su lado menor. ${ }^{27}$ Para comparar el efecto del PEA, la investigación se dividió en dos etapas: En la etapa previa al EA, que comprendió del 13 de marzo al 26 de mayo de 2000, se vigiló la conducta de los animales; durante este tiempo no se habían realizado actividades de EA y las jaulas eran sencillas (figura 1a). Esta fase se denominó etapa inicial (EI). La segunda fase del programa que ocurrió durante el EA comprendió del 26 de
Cuadro 3. Definición de categorías conductuales analizadas

\begin{tabular}{ll}
\hline $\begin{array}{l}\text { Categoría } \\
\text { conductual }\end{array}$ & \multicolumn{1}{c}{ Definición } \\
\hline Agresión & $\begin{array}{l}\text { Empujar, jalar, manotear, morder, prender, perse- } \\
\text { guir a otro individuo con la intención de infligirle } \\
\text { algún daño. Autoagresión: comportamiento que } \\
\text { implique una lesión a sí mismo. }\end{array}$ \\
Estereotipias & $\begin{array}{l}\text { Movimientos repetitivos que ocurren sin atender lo } \\
\text { que sucede alrededor como jalar el pelo, pellizcar } \\
\text { el cachete y balancear el cuerpo. }\end{array}$ \\
Exploración & $\begin{array}{l}\text { Observar, tocar y manipular objetos que se en- } \\
\text { cuentran en el entorno. }\end{array}$ \\
Coprofilia & $\begin{array}{l}\text { Tocar, oler, observar y/o embarrar intencional- } \\
\text { mente excremento u orina (no necesariamente el } \\
\text { propio), ya sea con la mano o con la boca. }\end{array}$ \\
Juego & $\begin{array}{l}\text { Prender, zarandear, perseguir y tocar a otro indi- } \\
\text { viduo o algún objeto sin hacerle daño. }\end{array}$ \\
\hline
\end{tabular}

noviembre de 2001 al 22 de marzo de 2002. Los monos estuvieron en contacto con las modificaciones hechas a las jaulas (figura 1b) y se realizaron las actividades del PEA; esta fase se denominó etapa experimental (EE).

Los tipos de EA contemplados en el programa fueron: enriquecimiento físico, enriquecimiento alimenticio e introducción de objetos novedosos. Con el apoyo del Proyecto 25858-H de CONACYT, las jaulas fueron enriquecidas físicamente al añadírseles diferentes estructuras que se muestran en la figura $1 b$. Como parte de la introducción de objetos novedosos y el enriquecimiento alimenticio se realizaron 11 actividades diferentes (cuadro 2), que se repitieron tres veces cada una. Cada semana se realizaron tres actividades diferentes seleccionadas al azar, en diferentes días, hasta completar 33 sesiones. Las actividades se programaron entre las 10:00 y las 16:00 horas.

El efecto del PEA sobre el bienestar de los animales fue evaluado por un observador con ocho meses de experiencia, quien realizó registros conductuales de las siguientes categorías: agresión, coprofilia, estereotipias, exploración y juego (cuadro 3).

Se realizaron registros continuos de 60 minutos utilizando un muestreo focal por conducta, ${ }^{28}$ en ambas fases de la investigación. Los registros fueron entre las 8:00 y las 19:00 horas y para cada etapa se obtuvieron 40 horas de registro. Durante la EE se realizaron estos registros cinco días a la semana, sin importar que hubiera o no actividad de EA.

\section{Análisis estadístico}

Para comparar estadísticamente la frecuencia de las conductas emitidas de una fase a otra, se aplicó la prueba no paramétrica de signos de Wilcoxon, ${ }^{29}$ con un nivel de significancia de $\mathrm{p} \leq 0.05$.

Cada categoría conductual y cada uno de los sexos se analizaron de forma independiente. Para realizar los cálcu- 
los estadísticos se utilizó el programa SPSS versión 17.0 para Windows (SPSS Inc. Chicago, IL. U.S.A.).

\section{RESULTADOS}

Los resultados muestran que, en la colonia de siete individuos, la agresión, las estereotipias y la coprofilia disminuyeron en frecuencia durante la EE. En las dos primeras categorías conductuales la reducción tiende a ser estadísticamente significativa (agresión, $\mathrm{N}=7, \mathrm{z}=-1,841$, $\mathrm{p}=0.063$; estereotipias, $(\mathrm{N}=7, \mathrm{z}=-1.521, \mathrm{p}=0.078)$. Por otro lado, la exploración y el juego $(\mathrm{N}=7, \mathrm{z}=-2.366, \mathrm{p}=0.008$ y $\mathrm{N}=7, \mathrm{z}=-2.214, \mathrm{p}=0.016$, respectivamente) fueron significativamente más frecuentes en la EE (figura 2).

Para conocer el efecto del EA sobre cada uno de los sexos, se aplicó la prueba no paramétrica de signos de Wilcoxon. La agresión fue menos frecuente tanto en machos como en hembras durante el EA sin significancia estadística (figura 3) (nMachos=3, $\mathrm{z}=-0.816, \mathrm{p}=0.375$; $\mathrm{nHembras}=4$, $z=-1.342, p=0.250)$; los machos son los que agreden más que las hembras en las dos etapas. En ambos sexos se observó un decremento de la coprofilia (nMachos=3, $\mathrm{z}=-1.342$, $\mathrm{p}=0.250$;
nHembras $=4, \mathrm{z}=-0.365, \mathrm{p}=0.438)$, y la reducción de las estereotipias tiende a ser significativa en las hembras (nMachos=3, $\mathrm{z}=-0.535, \mathrm{p}=0.375$; $\mathrm{nHembras}=4, \mathrm{z}=-1.461, \mathrm{p}=0.063$ ). La exploración fue más frecuente durante el EA en ambos sexos. Para las hembras, el cambio tiende a ser estadísticamente significativo con respecto a la EI (nMachos=3, $z=-1.604, p=0.125$; $\mathrm{nHembras}=4, \mathrm{z}=-1.826, \mathrm{p}=0.063)$. Finalmente, en la categoría de juego, tanto en los machos como en las hembras hubo un incremento en la frecuencia durante la EE sin que fuera significativo (nMachos=3, $z=-1.633, p=0.125$; nHembras $=4, z=-$ $1.633, \mathrm{p}=0.125)$ y se observó que los machos jugaron con más frecuencia que las hembras en ambas etapas del programa.

\section{DISCUSIÓN}

El EA consiguió que disminuyeran las conductas agresivas, estereotipadas y coprofílicas, mientras que se incrementó la frecuencia de las conductas de exploración y de juego, por lo que podemos decir que el programa de EA aplicado fue exitoso.

El hecho de no haber encontrado diferencias en la exploración entre las hembras puede deberse a que los tres machos monopolizaron los objetos. Habrá que realizar nuevos
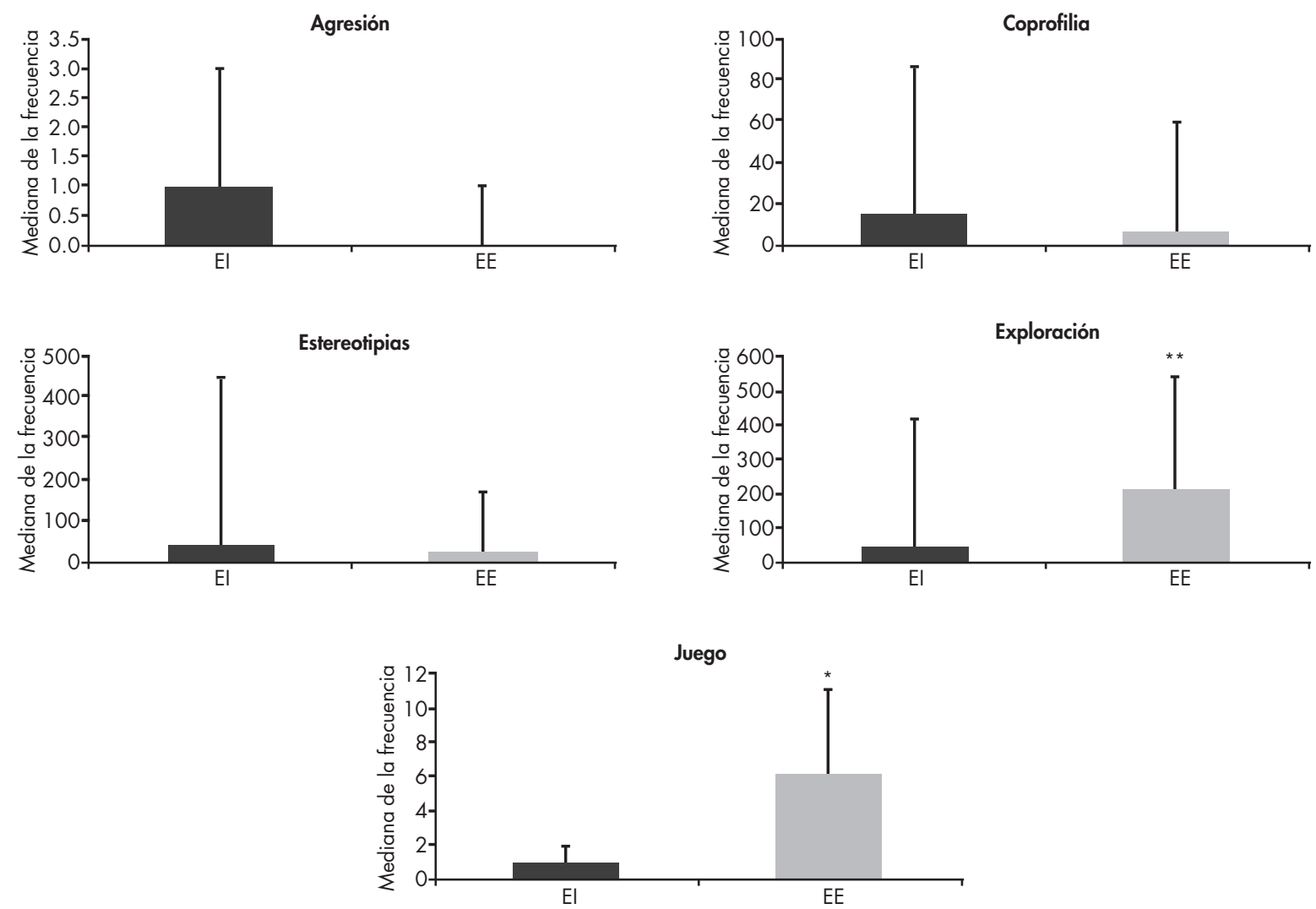

Figura 2. Comparación de la frecuencia de las categorías conductuales analizadas en ambas etapas del enriquecimiento ambiental. Mediana de la frecuencia y rango de cada categoría conductual analizada en la etapa inicial (El) y en la etapa experimental (EE) del enriquecimiento ambiental. $\left({ }^{*}\right)$ indica que $p \leq 0.05\left({ }^{*}\right)$ indica que $p \leq 0.01$. 

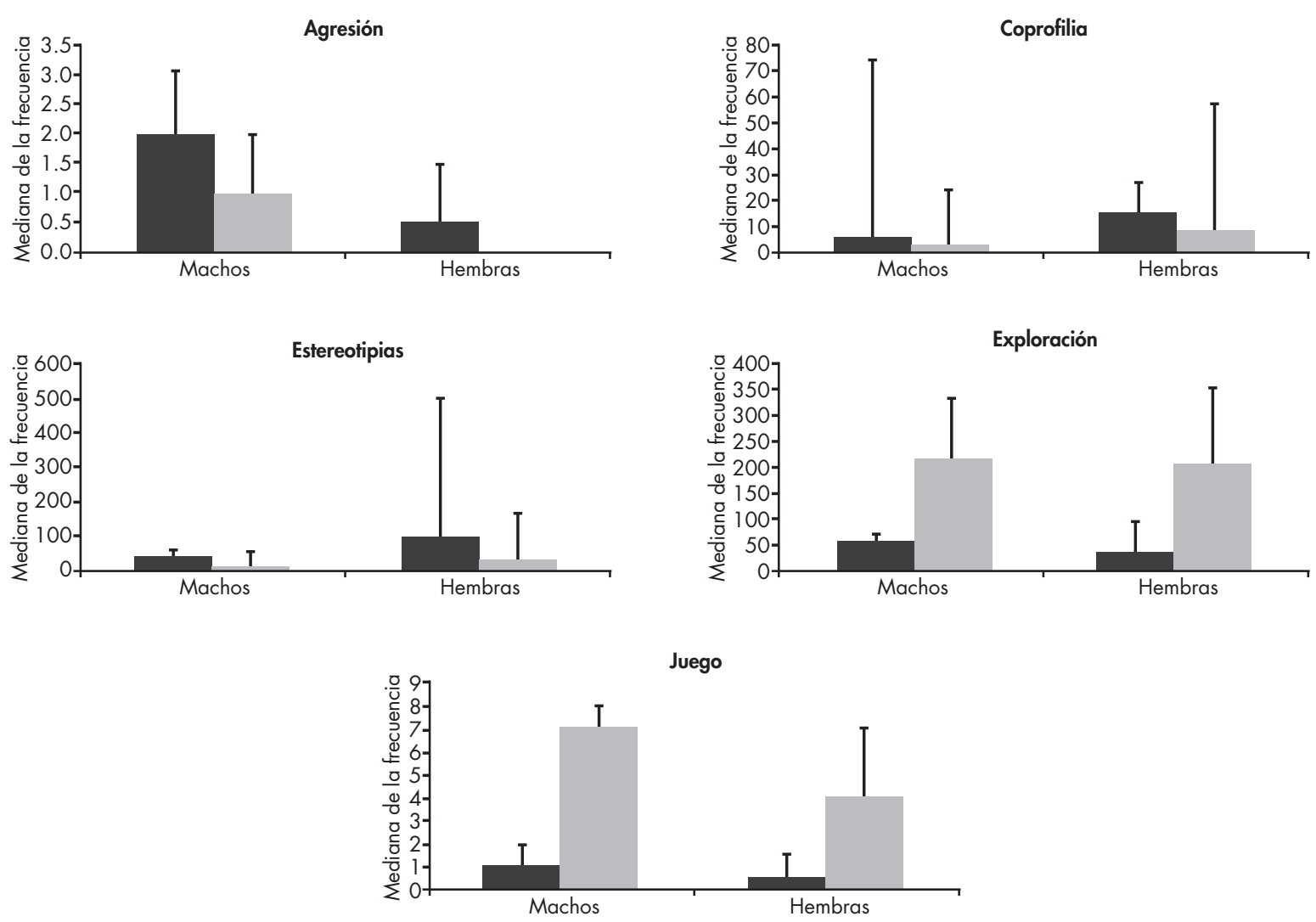

Figura 3. Comparación de la frecuencia de las categorías conductuales analizadas en ambas etapas del enriquecimiento ambiental según el sexo de los individuos. Mediana de la frecuencia y rango de cada categoría conductual analizada en la etapa inicial (EI) y en la etapa experimental (EE). Barras obscuras= etapa incial, barras claras= etapa experimental.

experimentos incrementando la disponibilidad de objetos. Con respecto al incremento del juego en los machos, aunque no fue significativa la diferencia con respecto a las hembras, se puede sugerir que esta conducta permite a los machos integrarse al grupo y posicionarse dentro de la jerarquía. ${ }^{30}$

Para proporcionar cualquier actividad de EA es fundamental considerar la especie con la que se trabaja, ya que de esto depende la respuesta de los individuos, pues las estructuras, las dinámicas y las interacciones difieren de una especie a otra. Sin embargo, dadas las serias condiciones en las que se encontraban los monos araña de este estudio, fue urgente aplicar un PEA general para esta colonia y simultáneamente para una colonia de macacos cola de muñón (Macaca arctoides) que también se encontraba en el Instituto Nacional de Psiquiatría. ${ }^{19}$ Por lo anterior, las actividades eran dirigidas a atender las necesidades de ambas especies, observando que los animales de ambas colonias presentaron mejoría en su comportamiento con las actividades. Las experiencias que se observaron durante los registros pueden ser consideradas en futuros trabajos. Actividades como "comida en el techo", "comida en botes" y "sonajas" provocaron que los monos se desplazaran y transportaran los objetos por la jaula; con las actividades "tronco" y "rodillo", los monos permanecieron atentos por la rareza de la situación. Por otro lado, se esperaba que actividades como recoger semillas del suelo podría dificultárseles a los monos araña por su anatomía, ya que en esta especie el dedo pulgar se ha reducido para facilitar la braquiación. ${ }^{31}$ Sin embargo, encontramos que la novedad de la actividad y la recompensa de poder comer las semillas alentó a estos animales y en consecuencia dedicaron tiempo a esta actividad. Además, se observó que los monos araña se acercaban y manipulaban inmediatamente los objetos y al cabo de 30 o 45 minutos ya no mostraban interés. Lo anterior es similar a lo reportado por Torstensson, ${ }^{26}$ quien menciona que el interés de los monos araña se pierde cuando la comida se termina. Por esto, es importante cambiar las actividades, los objetos, la hora y la forma en que éstos se presentan para mantener activos a los individuos.

El propósito de este trabajo fue proporcionar un entorno físico novedoso que despertara el interés de los animales, por lo que las actividades de enriquecimiento ambiental se enfocaron en enriquecer la jaula y ofrecer diferentes actividades de alimentación e introducción de objetos novedosos. Conocemos que en los programas de EA es necesario considerar el entorno social, ya que éste es muy importante para 
los primates. Asimismo, hay que tener en cuenta que, toda vez que sea posible, el hecho de tener contacto con otros individuos mejora el comportamiento, ${ }^{32}$ aunque en este caso no fue necesario implementar estrategias de enriquecimiento social, ya que los animales vivían en grupo.

En el caso del EO en primates humanos es necesario considerar las características físicas y mentales de los individuos, los aspectos sociales, políticos y culturales de la población para que las actividades sean aprovechadas al máximo. No olvidemos que por sí mismas las cárceles o los hospitales son pobres físicamente y que tal vez por la situación de reclusión no es posible mejorarlos. Sin embargo, es fundamental asumir que estas personas merecen mejores condiciones de vida, que en el caso de los prisioneros permitan su rehabilitación la cual debe ser el objetivo durante su estancia. La falta de actividades implica un detrimento en las habilidades sociales de los individuos e influye en su reintegración a la sociedad. El EO ofrece alternativas para los presos y favorece el crecimiento individual, social y cultural.

Es de especial interés desarrollar programas de EA en modelos primates no humanos a más largo plazo para obtener información relevante que contribuya a entender los procesos de pérdida de la libertad en primates.

\section{AGRADECIMIENTOS}

Agradecemos la ayuda de Pilar Chiappa con los análisis estadísticos. Esta investigación se realizó bajo los lineamientos de la Norma Oficial Mexicana NOM-062-ZOO-1999: Especificaciones técnicas para la producción, cuidado y uso de los animales de laboratorio.

\section{REFERENCIAS}

1. Shea SJ. Personality characteristics of self-mutilating male prisoners. J Clin Psychol 1993;49(4):576-585.

2. Dickie L. Environmental enrichment for old world primates with reference to the primate collection at Edinburg Zoo. Int Zoo Yb 1998;36:131-139.

3. Don Horner R. The Effects of an Environmental "Enrichment" Program on the Behavior of Institutionalized profoundly retarded children. J Appl Behav Anals 1980;13(3):473-491.

4. Mason GJ. Stereotypies: a critical review. Anim behav 1991;41:1015-1037.

5. O'Neill P. Developing effective social and environment enrichment strategies for macaques in captive groups. Lab Animal 1988;17(4):23-36.

6. O'Neill-Wagner P. Expression of species-specific behavior in rhesus monkeys. Lab anim 1994;23(10):48-44.

7. Kulpa AJ, Taylor S, Adams KM. USDA perspectives on environmental enrichment for animals. LAR Journal 2005;46(2):83-94.

8. Brüne M, Brüne-Cohrs U, McGrew W, Preuschoft S. Psychopathology in great apes: concepts, treatment options and possible homologies to human psychiatric disorders. Neurosci Biobehav Rev 2006;30:1246-59.

9. Chamove AS. Environmental enrichment: A review. Animal Techno$\operatorname{logy} 1989 ; 40(3): 155-178$.
10. Sacket G. The human model of psychological well-being in primates. En: Through the Looking Glass. Novak M, Petto A (eds.). Washington, DC: American Psychological Association; 1991.

11. Chang T, Forthman D, Maple TL. Comparison of confined mandrill (Mandrillus sphinx) behavior in traditional and "ecologically representative" exhibits. Zoo Biol 1999;18:163-176.

12. Lozano I. Manejo del comportamiento en primates cautivos mediante el uso de técnicas de enriquecimiento ambiental. Congreso Latinoamericano de Primatología del Nuevo Mundo. Colombia; 2001.

13. Lutz CK, Novak MA. Environmental enrichment for Nonhuman Primates: theory and Aplication. ILAR Journal 2005;46(2):178-191.

14. Vick SJ, Anderson J, Young R. Maracas for Macaca? Evaluation of three potential enrichment objects in two species of zoo-housed macaques. Zoo Biol 2000;19:181-191.

15. Bloosmith MA, Brent L, Schaprio SJ. Guidelines for developing and managing an environmental enrichment program for nonhuman primates. Lab Anim Sci 1991;41(4):372-377.

16. Carsltead K, Seidensticker J, Baldiwin R. Environmental Enrichment for Zoo bears. Zoo Biol 1991;10:3-16.

17. King CE. Environmental enrichment Is it for the birds? Zoo Biol 1993;12:509-512.

18. Brent L, Eichberg JW. Primate puzzleboard: A simple environmental enrichment device for captive chimpanzees. Zoo Biol 1991;10:353-360.

19. Márquez-Arias A, Santillán-Doherty AM, Arenas-Rosas RV, GascaMatías MP et al. Environmental Enrichment for Captive Stumptail Macaques (Macaca arctoides). J Med Primatol 2010;39:32-40.

20. Ghaziuddin N, McDonald C. A clinical study of adult coprophagics. Brit J Psychiat 1985;147:312-313.

21. Molineux ML, Whiteford GE. Prisons: From occupational deprivation to occupational enrichment. J Occupational Science 1999;6(3):124-130.

22. Bloosmith MA, Alford PL, Maple TL. Successful feeding enrichment for captive chimpanzees. Am J Primatol 1988;16:155-164.

23. Newberry RC. Environmental Enrichment: Increasing the biological relevance of captive environments. Appl Anim Behav Sci 1995;44:229-243.

24. Boccia ML, Laudenslager ML, Reite ML. Individual differences in macaques responses to stressors based on social and physiological factors: implications for primate welfare and research autocomes. Lab Anim 1995;29:250-257.

25. Cardona-López X, Zerda-Ordoñez E, Pérez-Torres J. Patrón comportamental y conductas estereotipadas de dos grupos cautivos de Ateles fusciceps robustus en Colombia. Universitas Scientiarum 2004;9:59-74.

26. Torstensson T. Enrichment for Colombian black spider monkeys (Ateles fusciceps rufiventris) in a zoo. 1irst cycle, G2E. Skara: SLU, Dept. of Animal Environment and Health. Suecia; 2009.

27. Ramírez MI, López AX. Distancias individuales en grupos cautivos de Macacos (Macaca arctoides). Tesis de Licenciatura. Biología. Facultad de Ciencias: UNAM. México; 1989.

28. Martín P, Bateson P. La medición del comportamiento. España: Alianza; 1991.

29. Siegel S. Estadística no paramétrica: aplicada a las ciencias de la conducta. $3^{a}$ edición. México: Trillas; 1990.

30. Wilson EO. Sociobiología. La nueva síntesis. Omega. España; 1980.

31. van Roosmalen MG, Klein LL. The spider monkeys, Genus Ateles. En: Mittermeier RA, Rylands AB, Colmbra-filho A, Fonseca GA (eds.). Ecology Bahavior Neotropical Primates. Washington, DC: Vol. 2; World Wildlife Fund; 1988.

32. Shanffner CM y Aureli F. Embraces and grooming in captive spider monkeys. International J Primatology 2005;26(5):1093-1106.

Artículo sin conflicto de intereses 